\title{
Vacuolated Lymphocyte to Leukocyte Ratio Measurement
}

National Cancer Institute

\section{Source}

National Cancer Institute. Vacuolated Lymphocyte to Leukocyte Ratio Measurement. NCI Thesaurus. Code C127627.

The determination of the ratio of vacuolated lymphocytes compared to total leukocytes present in a sample. The measurement may be expressed as a ratio or percentage. 